\title{
La tecnica videolaparoscopica in un programma di peritoneodialisi domiciliare
}

\author{
M. Giannattasio' ${ }^{1}$ R. La Rosa ${ }^{1}$, A. Balestrazzi ${ }^{2}$ \\ ${ }^{1}$ Unità Operativa di Nefrologia e Dialisi, \\ ${ }^{2}$ Divisione di Chirurgia, Presidio Ospedaliero Santa Maria degli Angeli, Putignano (Bari)
}

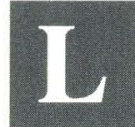

a videolaparoscopia (VL) ha avuto in questi ultimi anni un ampio utilizzo in campo chirurgico, rappresentando una evoluzione delle tecniche operatorie tradizionali; dapprima applicata a livello addominale per il trattamento della colelitiasi, successivamente è stata utilizzata in chirurgia ginecologica e toracica presentando gli innegabili vantaggi di una minore traumaticità dell'intervento, di una rapida ripresa della canalizzazione, della riduzione dei tempi di degenza e di cicatrici minime, consentendo di conseguenza una più rapida ripresa dell'attività lavorativa (Tab. I).

Scopo del lavoro è quello di valutare le possibilità di utilizzazione di tale tecnica in un programma di peritoneodialisi (PD) domiciliare.

\section{Materiali e metodi}

Il programma di PD domiciliare della Divisione di Nefrologia e Dialisi di Putignano comprende 22 pazienti (pz) uremici cronici terminali, che rappresentano il $37.9 \%$ della popolazione dialitica del centro.

Dal gennaio 1994 sono stati praticati 26 impianti di catetere peritoneale $(\mathrm{CP})$. L'impianto del CP viene routinariamente praticato dai nefrologi con la tecnica chirurgica di più diffuso impiego; si ricorre all'ausilio del chirurgo solo in particolari circostanze, quali: presenza di cicatrici addominali da pregressi interventi chirurgici (n. 7), presenza di grosso laparocele ipogastrico (n. 1), presenza di ernia inguinale (n. 1).

\section{TAB. I - VANTAGGI DELLA VL IN CHIRURGIA}

1. Minore traumaticità dell'intervento

2. Ridotta sintomatologia dolorosa post-intervento

3. Rapida ripresa della canalizzazione

4. Cicatrici minime

5. Risultato estetico migliore

6. Riduzione dei tempi di degenza

7. Più rapida ripresa dell'attività lavorativa

La VL è stata utilizzata in $8 \mathrm{pz}$, in $7 \mathrm{di}$ essi per l'impianto del CP, in $1 \mathrm{pz}$ per verificare le cause del malfunzionamento del CP dopo 24 mesi di PD.

Nei 7 pazienti nei quali è stata utilizzata la VL per l'impianto del CP, l'indicazione era stata la presenza di pregresse cicatrici addominali (n. 6) legate a pregresse laparotomie, quindi con rischio concreto di aderenze addominali e la necessità di praticare contemporaneamente una colecistectomia (CLx) (n. 1).

La tecnica videolaparoscopica già descritta in un nostro precedente lavoro (1), prevede in anestesia generale la creazione di un pneumoperitoneo mediante infissione dell'ago di Veress in zona di sicurezza, cioè lontano dalla cicatrice chirurgica, laddove presente; immessa la $\mathrm{CO} 2$ ed ottenuta una pressione endoperitoneale di $13 \mathrm{mmHg}$, si procede alla introduzione di un trocar da $10 \mathrm{~mm}$ in zona di sicurezza allo scopo di introdurre l'ottica laparoscopica connessa alla telecamera; si ispeziona l'interno della cavità addominale, per individuare il punto più favorevole per l'inserzione del $\mathrm{CP}$, che avviene attraverso un trocar da $5 \mathrm{~mm}$ introdotto in sede paramediana sotto diretto controllo visivo. Dopo aver ritirato la cuffia di dacron distale (diametro 4.9 $\mathrm{mm}$ ) appena al di sopra del peritoneo, nello spessore del muscolo retto addominale, si prova il funzionamento del $\mathrm{CP}$ 
infondendo la soluzione di lavaggio e, prima di estrarre il laparoscopio, si posiziona correttamente la punta del CP nello scavo di Douglas. Eseguite le manovre di tunnellizzazione sottocutanea dell'estremità prossimale del $\mathrm{CP}$, si crea l'exitsite a circa $3 \mathrm{~cm}$ dalla cuffia prossimale e si suturano con Vicryl 2-0 la fascia nel punto di introduzione del torcar da $10 \mathrm{~mm} \mathrm{e}$ con seta le ferite cutanee; il pronto recupero della soluzione dializzante testimonia la riuscita della procedura.

\section{Risultati}

La VL è stata utilizzata in 8 pz: in particolare, in $7 \mathrm{pz}$ per l'impianto del CP e in $1 \mathrm{pz}$ per accertare i motivi di una progressiva riduzione del funzionamento del CP (Tab. II).

L'inserzione del CP con VL è stata praticata a una pz (M.T. f/46 anni) da sottoporre a contemporanea CLx e a 6 pz con estese cicatrici addominali legate a pregresse laparotomie (n. 3 isterectomie, n. 2 appendicectomie, $n$. 1 colecistectomia).

In nessuno di questi pz è stato necessario lisare le aderenze, in quanto scarsamente rappresentate, eccetto che in una (N.L. $\mathrm{f} / 56$ anni) nella quale si è sezionata una lunga frangia omentale adesa al peritoneo parietale in quanto avrebbe potuto rappresentare ponte per strozzamento di volvolo interno. Nella pz A.M. (f/59) la VL è stata utilizzata a scopo diagnostico. Anamnesticamente si registrava: un intervento di appendicectomia all'età di 18 anni, l'impianto del CP pratico con tecnica chirurgica tradizionale in altro centro 24 mesi prima di giungere alla nostra osservazione. Durante il trattamento di PD la pz riferiva due episodi di peritonite e numerose dislocazioni del $\mathrm{CP}$. Al ricovero presentava ostruzione completa del CP con impossibilità sia di carico che di scarico del liquido dialitico, per cui veniva sottoposta ad Rx diretta addome che metteva in evidenza il normale posizionamento di un CP tipo coil ed assenza di distensione delle anse intestinali; successivamente un Rx-CP-grafia con iodamide metteva in evidenza una irregolare distribuzione del m.d.c. che in scopia appariva fuoriuscire dai primi forellini laterali del $\mathrm{CP}$. Le tradizionali manovre di disostruzione del CP risultavano infruttuose per cui in considerazione anche della indicazione alla CLx per colelitiasi si reputava opportuno procedere alla VL.

TAB. II - IMPIEGO DELLA VL IN UN PROGRAMMA DI PD

\begin{tabular}{|c|c|c|c|c|c|}
\hline $\mathrm{Pz}$ & Sesso/età & $\begin{array}{c}\text { Pregressa } \\
\text { laparotomia }\end{array}$ & Aderenza & CL & $\begin{array}{l}\text { Indicaz. } \\
\text { VL }\end{array}$ \\
\hline 1. N.L. & $\mathrm{f} / 56$ & isterectomia & +-- & -- & avvio PD \\
\hline 2. R.F. & $\mathrm{f} / 55$ & isterectomia & +-- & sì & avvio PD \\
\hline 3. R.D. & $\mathrm{f} / 73$ & appendicectomia & --- & -- & avvio PD \\
\hline 4. A.G. & $f / 84$ & colecistectomia & +-- & -- & avvio PD \\
\hline 5.M.T. & $f / 46$ & -- & --- & sì & avvio PD \\
\hline 6. A.P. & $\mathrm{f} / 69$ & isterectomia & --- & -- & avvio PD \\
\hline 7. G.D. & $m / 52$ & appendicectomia & +-- & -- & avvio PD \\
\hline 8. A.M. & $\mathrm{m} / 59$ & appendicectomia & ++++ & sì & ostruz. CP \\
\hline
\end{tabular}

TAB. III - INDICAZIONI ALLA VL IN PERITONEODIALISI

\section{Impianto del $\mathrm{CP}$ in $\mathrm{pz}$ con pregressi interventi laparotomici}

2. Necessità di interventi chirurgici eseguibili con tale tecnica

\section{Diagnosi e trattamento del malfunzionamento del $\mathrm{CP}$}

L'intervento metteva in evidenza la presenza di numerose aderenze tra anse intestinali, tra anse e peritoneo parietale, tra anse ed omento; tali aderenze interessavano molto più del $50 \%$ della superficie peritoneale totale, costituendo delle sacche chiuse in cui si è rinvenuto liquido sequestrato. Esse erano talmente estese da rendere difficoltoso l'accesso alla colecisti per la CLx ed in particolare hanno impedito l'esatta localizzazione del CP; tale situazione anatomica costituisce una controindicazione alla prosecuzione della PD (2). Si procedeva, pertanto, nel corso della stessa seduta operatoria alla rimozione del $\mathrm{CP}$ con tecnica tradizionale.

Uno dei 4 pz sottoposti a CLx videolaparoscopica ha presentato un dialisato effluente lievemente ematico della durata di 2 giorni. Il tempo impiegato in media per la procedura di semplice impianto del CP è stato di circa 30'. I pz dopo $8 \mathrm{~h}$ hanno assunto dieta liquida e hanno ripreso normale deambulazione. Per quelli avviati alla PD dopo un periodo di break-in di $14 \mathrm{gg}$ si è iniziato il trattamento depurativo; in nessun pz è stata necessaria una DP precoce.

\section{Discussione}

In questi ultimi anni si è assistito ad una rapida trasformazione delle tecniche operatorie grazie all'introduzione delle apparecchiature videolaparoscopiche in campo chirurgico. Dapprima applicata a livello addominale per il trattamento della colelitiasi la chirurgia videolaparoscopica si è estesa ad altri distretti addominali e successivamente anche a livello toracico. Tale tecnica è entrata nel bagaglio tecnico di molti chirurghi. Abbiamo, pertanto, pensato di valutare le possibilità di utilizzazione della VL in campo peritoneodialitico.

Nel nostro programma di PD la VL ha trovato le seguenti utilizzazioni: l'impianto del $\mathrm{CP}$, interventi di chirurgia VL e la diagnosi di malfunzionamento del CP (Tab. III).

L'impianto del CP con VL è stato praticato in $\mathrm{pz}$ che presentavano estese cicatrici addominali, conseguenza di pregressi interventi chirurgici. In tali pz esiste una maggiore difficoltà di posizionare il $\mathrm{CP}$ in sicurezza, senza cioè ledere incosapevolmente anse addominali adese al peritoneo perietale. Vi è, inoltre, la possibilità che, dopo l'impianto del CP con tecnica tradizionale, si abbiano per la presenza di estese aderenze intraperitoneali, problemi di recupero del dialisato e/o riduzione della superficie di scambio peritoneale, che potrebbero pregiudicare un adeguato trattamento dialitico. La VL presenta un basso rischio di lesione iatrogena delle anse intestinali in quanto il pneumoperitoneo praticato insufflando $\mathrm{CO}_{2}$ attraverso l'ago di Veress e l'inserzione dei trocar portante l'ottica si prati- 
cano a distanza di sicurezza dalla vecchia cicatrice. L'utilizzazione del trocar di Hasson, che si impianta in addome a cielo aperto, o l'adozione di particolari trocar che consentono di penetrare la parete addominale sotto il controllo visivo dell'ottica, possono ulteriormente ridurre il rischio di lesioni iatrogene. La visione diretta della cavità peritoneale permette di scegliere il punto più idoneo per l'introduzione del CP e pertanto di posizionarlo in maniera corretta; inoltre, è possibile l'immediata verifica del funzionamento del CP e quindi individuare le cause di un eventuale malfunzionamento. Un altro vantaggio è quello di valutare la presenza e l'entità delle aderenze peritoneali e quindi di praticare, in rapporto all'entità delle stesse, l'eventuale lisi; è, infatti, ben acclarato che allorquando la maggior parte della cavità peritoneale sia obliterata da aderenze, la lisi delle stesse e l'impianto del CP non siano indicate in quanto potrebbero vanificare l'efficacia della PD (2). Nei pz in studio l'entità delle aderenze era modesta tanto da non richiedere una lisi. La nostra esperienza in questi pazienti è stata incoraggiante e priva di inconvenienti a oltre 30 mesi dalla applicazione. La durata della procedura, se limitata al solo posizionamento del $\mathrm{CP}$ è più o meno simile alle altre tecniche e si aggira intorno a 20-30 minuti. Il costo della procedura che si svolge in narcosi ed in sala operatoria e che prevede il possesso e l'utilizzo della strumentazione videolaparoscopica, risulta indubbiamente più elevato e comporta la disponibilità di personale medico esperto nella tecnica e che possa far fronte tempestivamente ad ogni imprevisto, sempre possibile (Tab. IV).

Un'altra indicazione all'impianto di CP con VL può essere l'esecuzione di altro intervento chirurgico; in particolare l'intervento può essere eseguito contemporaneamente all'impianto del $\mathrm{CP}$, come nella nostra casistica, ovvero successivamente all'impianto cioè allorquando il pz sia già in trattamento di PD e necessiti di interventi di elezione praticabili con VL (3); in tali situazioni la DP può riprendere rapidamente senza il ricorso temporaneo alla emodialisi.

Altra indicazione è la diagnosi ed il trattamento del malfunzionamento del CP. Il caso da noi trattato conferma che la VL si impone sempre dopo l'insuccesso delle tradizionali manovre di disostruzione, in quanto solo con tale tecnica è possibi-

TAB. IV - VANTAGGI E SVANTAGGI DELLA VL PER L'IMPIANTO DEL CP
Vantaggi:
1. Basso rischio di lesioni iatrogene delle anse intestinali
2. Valutazione dell'estensione delle aderenze
3. Lisi delle aderenze (dove indicata)
4. Posizionamento esatto del CP sotto controllo visivo
5. Verifica immediata del funzionamento del $\mathrm{CP}$

\section{Svantaggi:}

\section{Anestesia generale \\ 2. Costi}

le diagnosticare con esattezza le cause del malfunzionamento ed effettuare l'eventuale correzione terapeutica laddove situazioni patologiche intraddominali non precludano la prosecuzione del trattamento PD. Repetto et al (4) hanno utilizzato tale tecnica principalmente nella diagnosi e nella terapia delle dislocazioni del CP. Nel nostro programma di PD abbiamo registrato un solo caso di dislocazione del CP regredito rapidamente con terapia medica.

In conclusione la VL, nella nostra esperienza, trova in un programma di PD domiciliare una valida collocazione tra le tecniche di impianto del CP, non come tecnica di routine ma specificatamente in $\mathrm{pz}$ che presentano estese cicatrici addominali da pregresse laparotomie per il rischio concreto di aderenze e possibilità di ledere inconsapevolmente anse intestinali adese al peritoneo parietale; inoltre, la VL può essere utilizzata per eseguire interventi di chirurgia addominale che se condotti con tecnica tradizionale non permetterebbero la ripresa immediata della PD. La VL accanto a innegabili vantaggi presenta, tuttavia, lo svantaggio di dover essere condotta da chirurgo esperto in sala operatoria ed in anestesia generale, quindi con costi sicuramente più elevati. Pertanto riteniamo che per la buona riuscita di un programma di PD sia indispensabile la stretta collaborazione con un chirurgo collaborante ed esperto di tale tecnica. Di fondamentale importanza è, inoltre, l'utilizzazione della VL nel malfunzionamento del CP in quanto permette di fare una esatta diagnosi e di essere utilizzata, laddove possibile, a scopo terapeutico per ripristinare la pervietà del $\mathrm{CP}$.

\section{BIBLIOGRAFIA}

1. Giannattasio M, De Maio P La Rosa R, Balestrazzi A. Videolaparoscopy: a new alternative for implantation of peritoneal catheters in ESRD patients with previous abdominal surgeries. Perit Dial Int 1996; 16: 96-7.

2. Twardowski ZJ. Peritoneal dialysis, current technology and techniques. Postgraduate Medicine. Peritoneal Dialysis 1989; 85: 161-82.

3. Mutter D, Marichal J, Heibel F, Marescaux J, Hannedouche T. Laparoscopy: an alternative to surgery in patients treated with continuous ambulatory peritoneal dialysis. Nephron 1994; 68: 334-7.

4. Repetto L, Boccardo E, De Prisco O, Donato G, Ettari G, Maurino D. La tecnica laparoscopica nella diagnostica e nella terapia di alcune complicanze in corso di dialisi peritoneale. Dialisi Peritoneale Atti del VII Congresso Nazionale: Wichtig Editore 1993; 533-7. 